\title{
Confocal Scanning Transmission Electron Microscopy: Theoretical Analysis of Three-Dimensional Imaging
}

\author{
J.J. Einspahr, P.M. Voyles
}

Materials Science and Engineering Department, University of Wisconsin-Madison, 1509 University Ave., Madison, WI 53706

Confocal microscopy is an imaging mode in which a pre-specimen lens focuses a fine probe on the specimen, then a post-specimen lens forms an image of the probe on an axial point detector. Frigo et al. have demonstrated the first confocal images with electrons, or confocal scanning transmission electron microscopy (STEM) [1]. They produced images of microelectronic circuits with resolution of $\sim 80 \mathrm{~nm}$ through a $5 \mu \mathrm{m}$-thick sample [1]. In optical confocal microscopy, 3-D images are routinely obtained by optical sectioning, which is stacking 2-D images acquired with different positions of the focal plane [2]. Optical sectioning with confocal STEM has only recently become useful with the advent of spherical aberration $\left(\mathrm{C}_{3}\right)$ corrected electron optics, but it holds potential for nanometer-resolution, 3-D imaging of nanostructures and macromolecules. We have investigated the potential 3-D imaging performance of current and next-generation aberration correctors and begun to investigate the limitations imposed by dynamical electron scattering in the sample.

We have calculated the optimum 3-D contrast transfer function (CTF) $H(l, w)$, where $l$ is the radial spatial frequency and $w$ is the spatial frequency along the optic axis, for confocal STEM based on the scalar wave theory of confocal imaging developed by $\mathrm{Gu}$ [2]. We have considered the effect of $\mathrm{C}_{3}$, fifth-order spherical aberration $\mathrm{C}_{5}$, and beam convergence semi-angle $\alpha$ for $200 \mathrm{kV}$ electrons with a point source and point detector. As in other imaging modes, it is possible to balance higherorder, un-adjustable aberrations (in this case $\mathrm{C}_{5}$ ) with lower-order, adjustable aberrations (in this case $\mathrm{C}_{3}$ ). Defocus is used to move the focal plane, so it has no effect on the 3-D CTF [2]. We define the optimum $\mathrm{C}_{3}$ for a given $\alpha$ as resulting in a minimum in the integrated difference between the aberrated CTF and the unaberrated CTF with same $\alpha$. Fig. 1 shows the optimum $\mathrm{C}_{3}$ versus $\alpha$ for $\mathrm{C}_{5}$ $=50 \mathrm{~mm} . \mathrm{C}_{3}$ scales as $\alpha^{2}$. As shown in Fig. 2(c), this $\mathrm{C}_{3}$ is not the same as that which minimizes the integrated aberration function $\chi(k)$. The optimum $\alpha$ is defined as the largest $\alpha$ which does not introduce severe distortions such as zero-transfer bands in the CTF; in practice this limits the integrated deviation between the aberrated and unaberrated CTF to $\sim 10 \%$.

For current-generation aberration correctors, we assumed $\mathrm{C}_{5}=50 \mathrm{~mm}$ [3]. The optimum conditions for a microscope equipped with two such correctors (one for the probe-forming lens and one for the image-forming lens) are $\mathrm{C}_{3}=-25.2 \mu \mathrm{m}$ and $\alpha=32.5 \mathrm{mrad}$. The 3-D CTF for a microscope with no aberrations and $\alpha=32.5 \mathrm{mrad}$ is shown in Fig. 2(a); the optimum aberrated CTF with $\mathrm{C}_{5}=50 \mathrm{~mm}$ is shown in Fig. 2(b). The aberrated CTF shows a dip in transfer at low spatial frequencies and an overall decrease in transfer at high axial spatial frequencies. Fig. 2(c) shows this more clearly in a cut through the 3-D CTF as a function of $w$ at $l=0.13 \mathrm{~nm}^{-1}$. Based on the CTF, this confocal STEM should achieve $2.4 \mathrm{~nm}$ axial resolution.

Our calculations also show that $1 \mathrm{~nm}$ axial resolution can be achieved in a confocal STEM with $\mathrm{C}_{5}=$ $4 \mathrm{~mm}$. Aberration correctors are under development that will correct $\mathrm{C}_{5}$, meeting this requirement. The conditions for $1 \mathrm{~nm}$ axial resolution are $\alpha=50.1 \mathrm{mrad}$ and $\mathrm{C}_{3}=-4.7 \mu \mathrm{m}$. 
We have also investigated whether dynamical electron scattering in the sample will make optical sectioning impossible through multi-slice simulations [4] of confocal STEM images. Simulated images of a $2 \mathrm{~nm}$ diameter gold nanoparticle embedded $9 \mathrm{~nm}$ deep in $18 \mathrm{~nm}$ of amorphous carbon with an unaberrated confocal STEM with $\alpha=25 \mathrm{mrad}$ do show optical sectioning, as shown in Fig. 3.

We will also discuss the limitations imposed by electron beam channeling on optical sectioning of zone-axis crystals and asymmetric lens configurations with only one aberration corrector.

\section{References}

[1] S. P. Frigo, Z. H. Levine and N. J. Zaluzec, Appl. Phys. Let. 81 (2002) 2112.

[2] M. Gu, Principles of Three-Dimensional Imaging in Confocal Microscopes, World Scientific, Singapore, 1996.

[3] N. Delby, O. L. Krivanek, P. D. Nellist, P. E. Batson, and A. R. Lupini, J. Electron Microscopy 50 (2001) 177.

[4] E. J. Kirkland, Advanced Computing in Electron Microscopy, Plenum Press, New York, 1998.

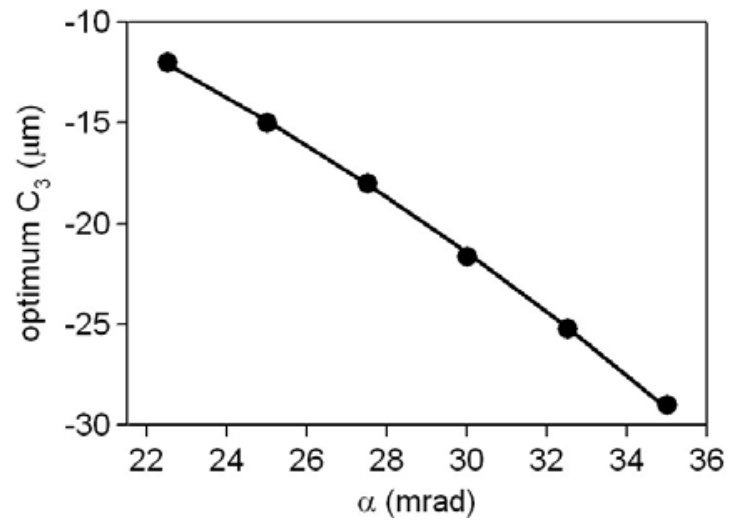

Fig. 1: Optimum $\mathrm{C}_{3}$ versus beam convergence semi-angle $\alpha$ for current aberration correction technology, $\mathrm{C}_{5}=50 \mathrm{~mm}$. $\mathrm{C}_{3}$ scales as $\alpha^{2}$.

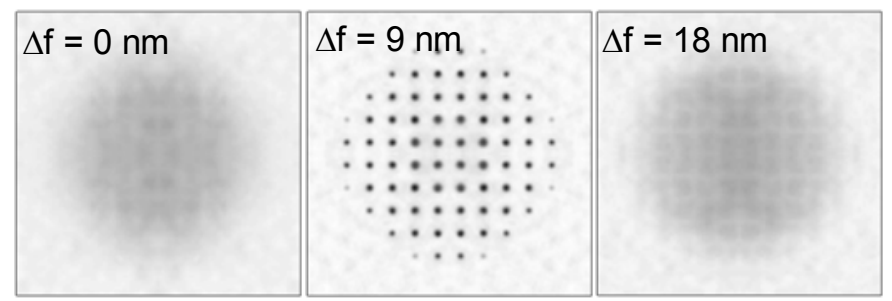

Fig 3: Multi-slice simulated confocal STEM images as a function of focal plane depth for an Au sphere 9 $\mathrm{nm}$ deep in $18 \mathrm{~nm}$ of amorphous carbon.
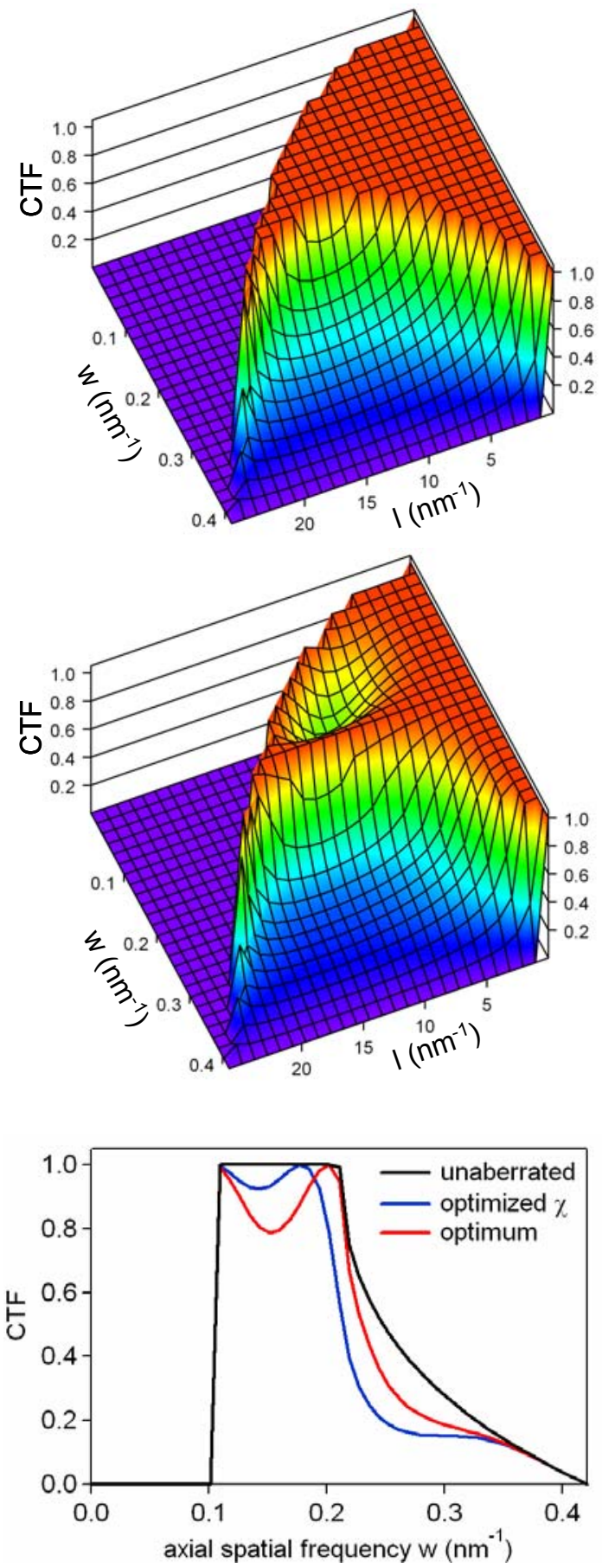

FIG. 2. CTF's for $\alpha=32.5 \mathrm{mrad}$ (a) without aberrations, (b) aberration compensated, $\mathrm{C}_{3}=$ $-25.2 \mu \mathrm{m}$ and $\mathrm{C}_{5}=50 \mathrm{~mm}$, and (c) CTF vs. $w$ at $l=1.3 \times 10^{10}$. 